\title{
Waste water microbiology
}

\section{Opinion}

The last decades have shown a reassessment of the issue of environmental pollution, under all aspects, both regionally and internationally. The progressive accumulation of organic compounds increasingly in natural waters is mainly due to the development and spread of chemical technologies and processing of organic synthesis. Population explosion, expansion of urban areas increased adverse impacts on water resources, especially in areas where natural resources are still limited. Currently, the use and reuse of water has become a major concern. Population growth leads to a significant increase in the volume of sewage default, making it an urgent imperative to develop effective and affordable technologies for treating wastewater. The physical-chemical processes common treatment using various chemical reagents and generates large quantities of sludge. The growing demand indicators of water quality regulations and drastic change in the elimination of waste water requiring the appearance and development of more efficient and effective processes. Each of these treatment methods has advantages and disadvantages. Exercises management of water resources increasingly urgent demands for wastewater treatment technologies to reduce the negative impact of the industry of natural water sources. Therefore, the new regulations and emission limits imposed and industrial activities are required to look for new methods and technologies capable of effectively removing loads of heavy metals pollution and reducing the volume of waste water, closing the water cycle, or by reusing and recycling waste water. Advanced technologies for wastewater treatment are needed to remove contamination and can increase the processes of destruction or separation of contaminants, such as advanced methods of oxidation, chemical precipitation, adsorption on various media, etc. These technologies can be applied successfully to remove contaminants partially removed by conventional methods, for example, biodegradable organic compounds, suspended solids, colloidal substances, and phosphorus and nitrogen compounds, heavy metals compounds dissolved, allowing the microorganisms that recycle wastewater was paid special attention to the electrochemical technologies because they have advantages: versatility, safety, selectivity, possibility of automation, environment and requires low investment costs technologies for treating wastewater containing organic compounds fall within one of the following categories:
Volume 2 Issue I - 2017

\author{
Maulin P Shah \\ Head-Microbiology Lab, Enviro Technology Limited, India
}

Correspondence: Maulin P Shah, Head-Microbiology Lab, Enviro Technology Limited, Davison of Applied \& Environmental Microbiology, India, Tel +91-9099965504,

Emailshahmp@beil.co.in

Received: February 10, 2017| Published: February 15, 2017

I. The non-destructive procedures: Based on the physical processes of adsorption, extraction, separation, etc.

II. Destructive biological processes: Based on biological processes using activated sludge.

III. Oxidative destructive processes: Processes based on chemical oxidants, which, in turn, may fall within one of the following categories:

a. Incineration.

b. Wet oxidation-which operates under high temperature and pressure versions.

c. Wet air oxidation-wet oxidation with $\mathrm{O}_{2}$ oxidant air.

d. Catalytic wet air oxidation- $\mathrm{O}_{2}$ oxidation catalytic wet air oxidizing agent.

e. Supercritical Water Oxidation-oxidation with $\mathrm{O}_{2}$ oxidant air in supercritical conditions Choosing the most suitable method of waste water treatment studies require both increased efficiency and economic efficiency in terms of investment and operating costs.

\section{Acknowledgements}

None.

\section{Conflict of interest}

Author declares that there is no conflict of interest. 